\title{
La calidad en la educación superior universitaria en tiempos de pandemia: una perspectiva estudiantil
}

\author{
Quality in university higher education in times of pandemic: A student perspective
}

Ketty Marilú Moscoso-Paucarchuco ${ }^{1}$, José Manuel Castillo-Custodio ${ }^{2}$, Manuel Michael Beraún-Espíritu ${ }^{3}$

\section{RESUMEN}

Objetivo: Medir la calidad en la educación superior universitaria en tiempos de pandemia, en cuatro universidades de la región Junín, durante el periodo 2021, desde una posición estudiantil. Material y métodos: Se empleó el método de investigación científico de tipo aplicado, nivel descriptivo, de diseño cuantitativo, de una sola variable; en una muestra de estudiantes variada de carácter no inferencial. Resultados: El estudiante universitario conoce los objetivos académicos de su universidad y que estos deben orientarse a la gestión de calidad; respecto a la oferta educativa, muchos ignorar su contenido; los servicios de infraestructura y mantenimiento se han detenido debido a la pandemia; los docentes realizan y ejecutan investigación científica, la misma que es publicada en los repositorios de cada institución; cumplen en mayoría con el perfil detallado en la Ley Universitaria 30220; los servicios complementarios deben traducirse a una forma virtual; bajo estas circunstancias la bolsa de trabajo es uno de los principales intereses del estudiantado: la transparencia muestra cierta debilidad, por falta de comunicación y socialización de decisiones tomadas por las autoridades universitarias. Conclusiones: La mayoría de estudiantes se encuentra satisfecho con los esfuerzos desarrollados para el cumplimiento de las actividades académicas en tiempos de pandemia.

PALABRAS CLAVE: Calidad, educación superior universitaria, pandemia, estudiantes.

\section{SUMMARY}

Objective: to measure the quality of university higher education in times of pandemic, in four universities in the Junín region, during the 2021 period, from a student position. Material and methods: The scientific research method of applied type, descriptive level, quantitative design, of a single variable was used; in a mixed sample of noninferential students. Results: the university student knows the academic objectives of his university and that these should be oriented to quality management; regarding the educational offer, many ignore its content; infrastructure and maintenance services have been stopped due to the pandemic; teachers carry out and execute scientific research, which is published in the repositories of each institution; Most of them comply with the profile detailed in University Law 30220; ancillary services must be translated into virtual form; Under these circumstances, the job market is one of the main interests of the student body: transparency shows a certain weakness, due to lack of communication and socialization of decisions made by university authorities. Conclusions: The majority of students are satisfied with the efforts made to fulfill academic activities in times of pandemic.

KEY WORDS: Quality, university higher education, pandemic, students.

\footnotetext{
Universidad Privada de Huancayo Franklin Roosevelt. Huancayo, Perú.

Universidad Peruana Los Andes. Huancayo, Perú.

Universidad Continental. Huancayo, Perú.
} 


\section{INTRODUCCIÓN}

La calidad en la educación superior universitaria ha sido preocupación constante en el país, este aseguramiento de la calidad fue delimitada por el Ministerio de Educación a través de VIII condiciones básicas de calidad que debe cumplir la institución educativa universitaria, accediendo gracias a este cumplimiento a la licencia institucional. Un sistema de educación superior, puede ser exitoso en pocos años si hay políticas públicas coherentes con la realidad social (1) y para ello, es importante involucrar a los stackholders (grupos de interés), principalmente a los estudiantes, quienes evalúan la calidad, manifestando satisfacción o insatisfacción en cada uno de los procesos misionales de la organización educativa (tabla 1).

El Ministerio de Educación controla los indicadores de calidad bajo ciertos estándares, condiciones que no son socializadas a la población estudiantil en su totalidad. La educación siempre ha tenido una prioridad central en todas las naciones del mundo, por ello se ha creado diversas herramientas de gestión para poder facilitar su acceso y mejorarla, llegando a brindar un servicio de calidad, que otorgue sostenibilidad a una nación.

En América Latina la autoevaluación y evaluación en las universidades, no ha sido una práctica común, como sucede en los países desarrollados. Hay poca experiencia en relación a las políticas y prácticas de evaluación, aunque recientemente existe una preocupación generalizada sobre los procesos de evaluación y acreditación que surge en el contexto de una crisis en las relaciones Universidad - Estado y Universidad - Sociedad (3). Las estrategias políticas del país, se direccionan a medir la calidad, desde un punto institucional.

Resulta importante medir la calidad de un servicio, recientemente existe un crecimiento en el interés por la calidad educativa en el ámbito universitario como consecuencia de las demandas sociales, políticas y económicas que exigen la participación activa de la universidad en el desarrollo de los países, por ello es necesario identificar cuáles son sus factores determinantes y cómo afectan el funcionamiento de la universidad (4). Para Guadarrama-González (2021)(5) la calidad en el nivel superior es la articulación entre ciencia, docencia y conciencia.

En la actualidad, Perú cuenta con 145 universidades, de las cuales 94 cuentan con licencia institucional y 51 presentan licencia denegada o se encuentran en plan de adecuación (tabla 2).

Las dimensiones de la calidad en la educación superior universitaria actualmente se encuentran prescritas en el Modelo de Licenciamiento y su implantación en el Sistema Peruano, se muestra solo a nivel institucional, para este estudio se ha adecuado los 55 indicadores al interés del estudiante y la realidad pandémica actual, resumiéndose en 30 interrogantes dicotómicas, la que brinda la oportunidad de analizar ciertas perspectivas respecto a la prestación del servicio educativo.

Tabla 1. Condiciones Básicas de calidad

\begin{tabular}{|c|c|}
\hline Condiciones & $\mathrm{N}^{0}$ de indicadores \\
\hline $\begin{array}{l}\text { Condición I: Existencia de objetivos acaémicos, grados y títulos a otorgar, y planes de estudios } \\
\text { correspondientes }\end{array}$ & 8 \\
\hline $\begin{array}{l}\text { Condición II: Oferta educativa a crease compatible con los fines propuestos en los instrumentos de } \\
\text { planeamiento }\end{array}$ & 7 \\
\hline $\begin{array}{l}\text { Condición III: Infraestructura y equipamiento adecuado al cumplimiento de sus funciones (aulas, } \\
\text { bibliotecas, laboratorios, entre otros) }\end{array}$ & 15 \\
\hline Condición IV: Líneas de investigación a ser desarrolladas & 8 \\
\hline $\begin{array}{l}\text { Condición V: Verificación de la disponibilidad de personal docente calificado con no menos de } 25 \% \text { de } \\
\text { docentes a tiempo completo }\end{array}$ & 4 \\
\hline $\begin{array}{l}\text { Condición VI: Verificación de los servicios educacionales complementarios básicos (servicio médico, social, } \\
\text { psicopedagógico, deportivo, entre otros) }\end{array}$ & 8 \\
\hline Condición VII: Existencia de mecanismos de mediación e inserción laboral (Bolsa de Trabajo u otros) & 4 \\
\hline Condición VIII: CBC Complementaria: transparecia de universidades & 1 \\
\hline Total & 55 \\
\hline
\end{tabular}

Nota. Detalle de las VIII Condiciones básicas de calidad con la cantidad de indicadores regulados en el ordenamiento jurídico peruano (2). 
Tabla 2.Condición de licenciamiento en universidades peruanas

\begin{tabular}{lr}
\hline \multicolumn{1}{c}{ Condición } & Cantidad \\
\hline Licencia otorgada & 94 \\
Licencia denegada & 47 \\
Plan de adecuación & 4 \\
Total & $\mathbf{1 4 5}$ \\
\hline
\end{tabular}

Nota. Detalle de número de universidades bajo concisión de licencia otorgada, denegada o en plan de adecuación. Adaptado de SUNEDU (2).

En la literatura académica, la medición de la calidad del servicio ha suscitado algunas diferencias de criterio. La principal hace referencia a qué es lo que realmente se está midiendo. En general se encuentran tres tendencias de constructos que se usan para evaluar la calidad del servicio: calidad, satisfacción y valor (6). En el caso de prestación de servicios se orienta a la medición de la calidad a través de las condiciones básicas, la satisfacción calculando lo esperado o por encima de lo esperado y la propuesta de valor diferenciada de cada universidad.

Según Newman, citado por Gonzáles "La universidad es el lugar en que se enseña el conocimiento universal. Esto implica que su objeto es, por una parte, intelectual, no moral; y por la otra, que es la difusión y extensión del conocimiento, más que el avance del conocimiento (7). Si su objeto fuese el descubrimiento científico y filosófico, yo no veo por qué una universidad debía tener estudiantes; si fuese el entrenamiento religioso, yo no veo cómo puede ser la casa de la literatura y de la ciencia". Estas instituciones se preocupan por una formación profesional tridimensional enmarcada en lo académico, investigativo y extensión social.

La educación de calidad es excelencia, basado en la definición tradicional, equivalente a poseer estudiantes sobresalientes, académicos destacados, y aseguramientos del primer nivel (8). Este pensamiento se puede aplicar a una educación de minorías que se refleje en estudiantes competentes y aptos para continuar de manera exitosa el nivel superior. La calidad educativa es definida a partir de un conjunto de especificaciones que deben ser cumplidas y cuyo grado de consecución puede ser medido objetivamente (9). Estos procedimientos de evaluación deben tener validez, y proponer significaciones alternas.

En el Perú, la definición adoptada en la Política de Aseguramiento de la Calidad de la Educación Superior
Universitaria es la referida a calidad como adecuación a propósitos; es decir, la calidad se define como el grado de ajuste entre las acciones que lleva a cabo la universidad o programa académico para implementar las orientaciones contenidas en la misión y propósitos institucionales, y los resultados que dichas acciones (10). Se considera que los propósitos institucionales tienen condiciones internas y externas, por una parte, la preparación académica, con competencias de innovación e investigación, la conciencia social y por otra parte la oportunidad de desenvolverse en un mercado laboral y un entorno socio económico, de manera ética y proba.

Conocida también como educación a distancia o educación e-learning, educación para apoyo a la presencialidad (b-learning), educación para móviles (m-learning) entre otras; todas estas metodologías buscan escenarios adecuados para apoyar la correcta operación del entorno virtual para el aprendizaje (EVA), los cuales buscan ambientar el ciberespacio, que codyuve el proceso metodológico para lograr una educación efectiva (11). En la educación virtual se aprecia la importancia de la gestión del conocimiento, su evolución y sus posibles limitaciones (12). Se dicta bajo una plataforma que no tiene la arquitectura física de un panóptico (13). En Perú la modalidad virtual se encontraba en proceso de iniciación antes de la pandemia, creciendo a paso agigantado con la reglamentación de educación no presencial.

En la virtualidad se busca que la organización cumpla ciertos requisitos para cubrir la necesidad o expectativa, en términos de educación de los estudiantes, con la finalidad de lograr calidad con escalonamiento hacia la excelencia (14). En el Perú, la definición adoptada por en la Política de Aseguramiento de la Calidad de la Educación Superior Universitaria es la referida a calidad como adecuación a propósitos; es decir, la calidad se define como el grado de ajuste entre las acciones que lleva a cabo la universidad o programa académico para implementar las orientaciones contenidas en la misión y propósitos institucionales, y los resultados que dichas acciones obtienen (10). Estas dimensiones se obligan a medirse de manera interna y externa, así como en función a los tres pilares que se asocian la parte académica, investigativa y de proyección social, basado en 8 indicadores de calidad, según (10) y 1 de satisfacción, parte del aporte en la investigación.

D1 = Objetivos académicos: definido por los programas de estudio, el perfil del graduado, objetivos 
institucionales, todos reflejados en herramientas de gestión debidamente aprobados.

D2 = Oferta educativa: previsiones económicas y financieras de la universidad, legalidad de las fuentes de financiamiento.

D3 = Infraestructura y equipamiento: ubicación y posesión de locales, seguridad estructural de laboratorios, talleres, disponibilidad de servicios públicos, ambientes y espacios de apoyo docente y estudiante.

D4 = Líneas de investigación: docentes que realizan investigación, registro de proyectos de investigación. D5 = Docentes calificados: experiencia docente, evaluación de desempeño, capacitación, cumplimiento de requisitos para ejercicio docente.

D6 = Servicios complementarios: de salud, social, psicopedagógicos, deportivos, culturales, seguridad y vigilancia.

D7 = Bolsa de trabajo: mecanismos de inserción laboral para estudiantes y egresados.

D8 = Transparencia: información institucional en el portal web, admisión, gestión de la calidad, procesos administrativos automatizados.

D9 = Satisfacción: en lo académico, investigativo y proyección social.

\section{MATERIAL Y MÉTODOS}

La investigación es científica, de tipo aplicada, no experimental, observacional, prospectivo. El nivel de investigación es descriptivo. El diseño de la investigación es cuantitativa, inductiva deductiva, de carácter no inferencial. El estudio de la variable se desarrollará de acuerdo al siguiente procedimiento:

- Validación del instrumento por expertos.

- Determinación de la muestra por conveniencia o por oportunidad de las universidades detalladas en la población.

- Aplicación del instrumento cuestionario a través de formulario google, mediante comunicación virtual.

- Procesamiento de la información a través de herramienta de Office-Excel.

- Comparación de resultados de las muestras estudiadas de las diversas universidades de la región Junín.

La población son los estudiantes de las diversas universidades con licencia institucional de la Región Junín. Señalando dentro de ellas a la Universidad Nacional del Centro del Perú, la Universidad Peruana los Andes, la Universidad Continental y la Universidad Roosevelt, en adelante UNCP, UPLA, UContinental y URoosevelt. Al tener cuatro fuentes de población la muestra fue variada y se determinó durante el proceso de investigación, la muestra de tipo fundamental, por oportunidad o conveniencia favorecerá el desarrollo del estudio de carácter cualitativo, estas muestras están formadas por los casos disponibles a los cuales tenemos acceso. Esta decisión se puede tomar antes y después de la recolección de los datos, eso es relativo debido a que el proceso cualitativo es iterativo y emergente (15).

La elección fue propositiva o de juicio, no parte de un número determinado de antemano (16) . Como con toda contundencia lo declara Patton12, en este campo no hay reglas para decidir el tamaño de la muestra $\mathrm{y}$, si hubiera que enunciar alguna, está sería: "todo depende". Depende del propósito del estudio, de lo que resulta útil para lograrlo, de lo que está en juego, de lo que lo hace verosímil, y en última instancia, incluso de lo que es posible (17). Según HernándezSampieri (18), las muestras utilizadas con frecuencia en investigaciones regionales o locales, según el área de estudio es de 100 (a nivel regional), por lo que se opta por la siguiente muestra, la misma que escogida por oportunidad o conveniencia.

De acuerdo al análisis de consistencia y fiabilidad del instrumento, el mismo que se encuentra determinado por escalas, resulta un valor de 0,754 ; lo que significa que el instrumento de investigación es bueno. En el procedimiento de comparación de los resultados de las cuatro universidades se utilizará el Office- Excel y criterio interpretativo. Los instrumentos de acuerdo a cada una de las técnicas serán: fichas de contenido, fichas bibliográficas, fichero y cuestionario. La validación del instrumento será a juicio de expertos. La validación del instrumento se determinó bajo la técnica del Alfa de Cronbach, para lo cual se recurrió a tres profesionales con grado de magister, conocedores de la docencia universitaria (tabla 3).

Tabla 3.Resultado de Alfa de Cronbach

\begin{tabular}{lr}
\hline \multicolumn{1}{c}{ Alfa crombach } & $\mathbf{0 , 7 5 4}$ \\
\hline $\mathrm{k}$ & 10 \\
sum Var Ítems & 3 \\
Var totales & 9,333333333 \\
Sección 1 & 1,111111111 \\
sección 2 & 0,678571429 \\
\hline Nota. Valorización de ítems y Alfa de Cronbach
\end{tabular}


Los resultados son estrictamente usados para términos académicos e investigativos, se busca solucionar problemas de entorno social, no existiendo aspecto ético contradictorio de ningún tipo.

\section{RESULTADOS}

Análisis de la Dimensión 1 (D1) Objetivos académicos.

Dentro de los objetivos académicos se plantean 6 ítems (P), las que se consideran generan mayor interés en la población estudiantil.

Respecto a la P01. ¿Conoce los objetivos institucionales de su universidad? el 54\% respondió que sí; respecto a la P2. ¿Conoce el plan de estudios de su carrera? el 95\% respondió que sí; en la P3.
¿Conoce la reglamentación para la obtención del bachiller y título? 63\% respondieron positivamente; en la P4. ¿Utiliza el sistema de su universidad para solicitar información, realizar trámites, matrículas, consulta de registro académico, entre otros? 65\% respondieron afirmativamente; en la P5. ¿Sabe si su universidad ha convocado últimamente a un proceso de admisión? 62\% respondieron que sí; en la P6. ¿Tiene conocimiento respecto a las labores que realiza el área de gestión de la calidad? sólo el $27 \%$ respondieron que sí (grafico 1).

Las universidades materia de muestra para la investigación respecto a la dimensión de objetivos académicos presentan cierta fortaleza, salvo en los trabajos que se realizan a nivel de gestión de calidad, donde presenta mayor debilidad y presencia, ya que

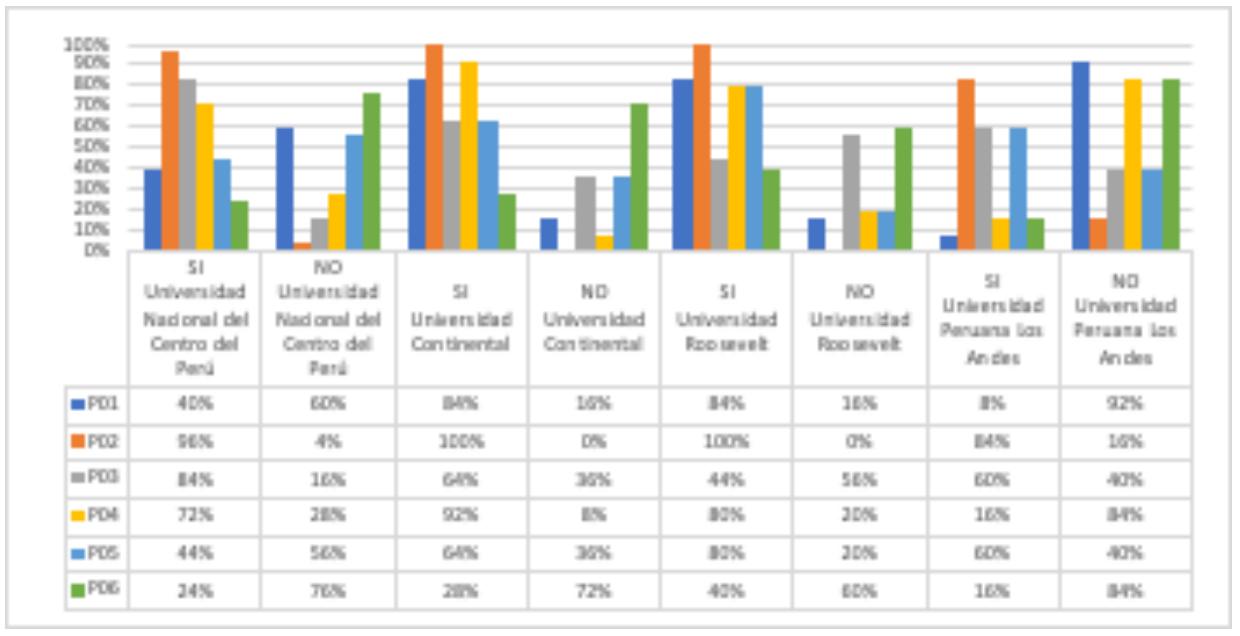

Nota: Dimensión 1, ítems de 1 a 6.

Gráfico 1. Análisis de la Dimensión 1 (D1) Objetivos académicos.



Nota. Dimensión 2, ítem 7.

Gráfico 2.Análisis de la Dimensión 2 (D2) Oferta educativa. 
todos los procesos deben someterse a altos estándares de evaluación.

Análisis de la Dimensión 2 (D2) Oferta educativa

En el análisis de la dimensión 2 Oferta educativa, de un solo ítem, la P07. ¿Tiene conocimiento si su universidad creará nuevas escuelas o carreras profesionales?, al respecto $77 \%$ de los estudiantes respondieron que desconocen de tal información (gráfico 2).

Análisis de la Dimensión 3 (D3) Infraestructura y equipamiento

En la dimensión 3 Infraestructura y equipamiento, podemos observar en el gráfico 3 del análisis de la P8. ¿Su universidad ha reemplazado los servicios de infraestructura y equipamiento por servicios virtuales (simulación de laboratorios, bibliotecas virtuales)? $57 \%$ los estudiantes respondieron positivamente; se podría determinar que se ha suplido los servicios de infraestructura.

Análisis de la Dimensión 4(D4) Líneas de investigación

Según el gráfico 4, se analiza la P09. ¿Sabe si sus docentes realizan investigación científica? 62\% respondió que sí; analizando la P10. ¿Ha visitado la página del repositorio institucional de su universidad? $51 \%$ de los estudiantes respondieron sí; en la P11. ¿Actualmente docentes y estudiantes ejecutan proyectos de investigación? 79\% los estudiantes afirman su respuesta. Se determina que la mayoría de los estudiantes de la Región Junín saben que sus docentes realizan y ejecutan investigación científica y que la gran mayoría ha visitado los repositorios de sus universidades.

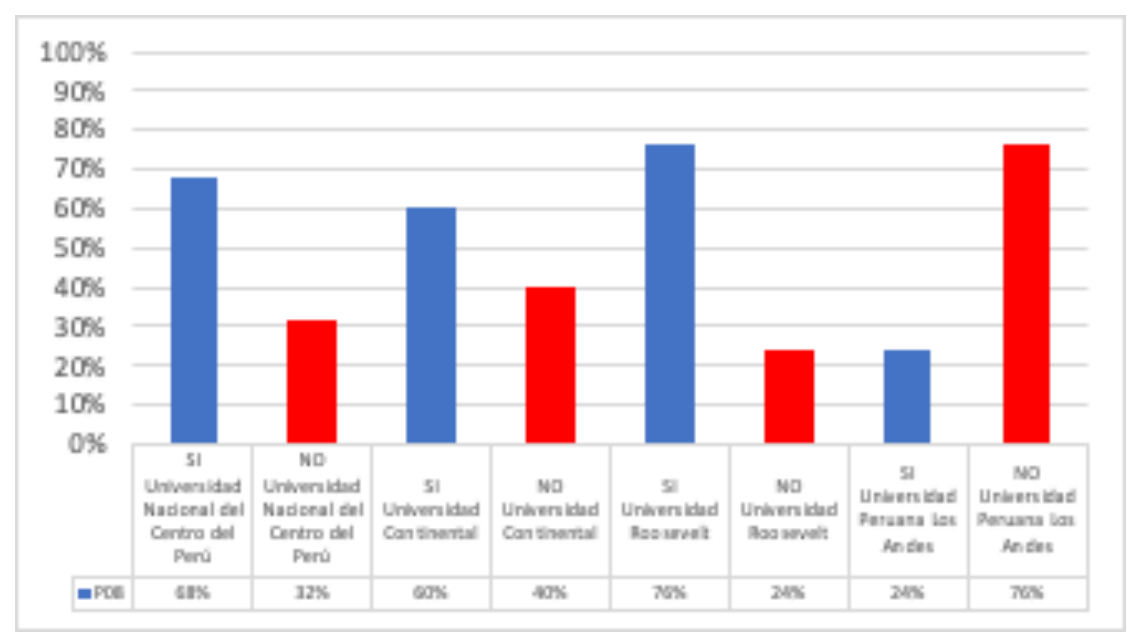

Nota. Dimensión 3, ítem 8.

Gráfico 3. Análisis de la Dimensión 3 (D3) Infraestructura y equipamiento.

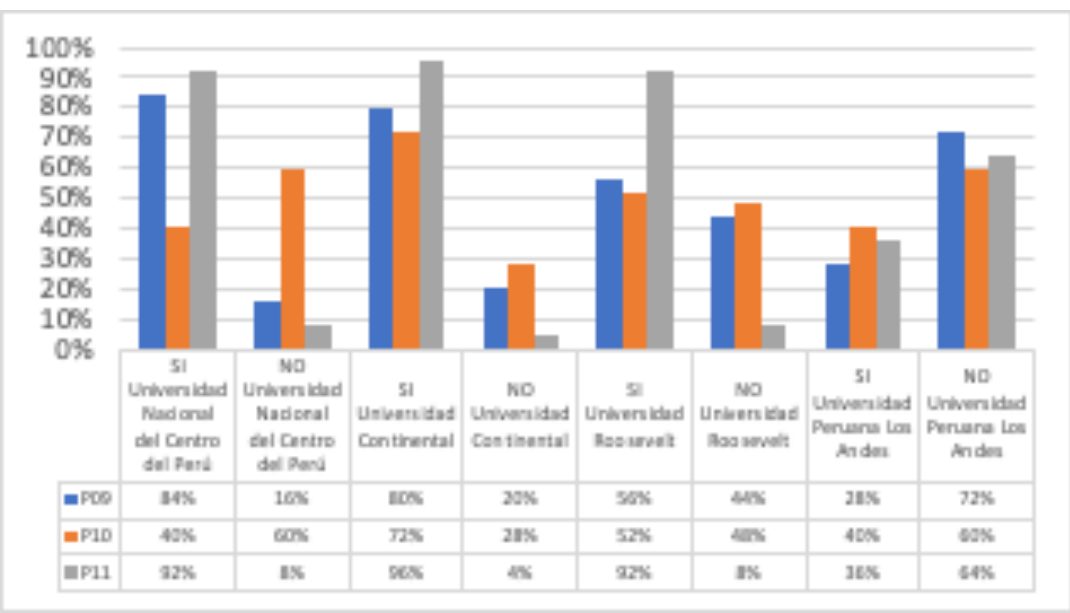

Nota. Dimensión 4, ítems de 9 a 11.

Gráfico 4. Análisis de la Dimensión 4 (D4) Líneas de investigación 
Análisis de la Dimensión 5 (D5) Docentes calificados

En el gráfico 5, se revisa la P12. ¿Cuenta con docentes que tienen grado de maestría o doctorado? dentro de las respuestas el $79 \%$ de los estudiantes dice que no; en la P13. ¿Está enterado de concursos de selección y evaluación docente? Ante esta situación de educación virtual la gran mayoría de estudiantes $(52 \%)$ no se encuentra informado respecto a la selección y evaluación docente; en la P14. ¿Tiene conocimiento si su universidad capacita a sus docentes?, $58 \%$ de los estudiantes respondieron que sí conocen de los esfuerzos de sus universidades en la realización de capacitación docente.

Bajo estos indicadores se puede manifestar que se necesita desarrollar la comunicación a los estudiantes respecto a los concursos y evaluación de los docentes, así como a las capacitaciones de los mismos que realizan estos entes educativos. El cumplimiento de los requisitos mínimos de la Ley Universitaria para el ingreso a la carrera docente es de vital importancia.

\section{Análisis de la Dimensión 6 (D6) Servicios complementarios}

Respecto a la P15. ¿Su universidad ofrece servicios de salud de manera virtual? el 50\% respondió que sí, en la P16. ¿Su universidad ofrece servicios sociales de manera virtual? el 56\% afirmaron que su universidad adecuó los servicios a la modalidad virtual; ante la P17. ¿Su universidad ofrece servicios psicopedagógicos de manera virtual? 56\% respondieron positivamente, a la P18. ¿Su universidad ofrece servicios deportivos (ajedrez, u otro) de manera virtual? 51\% respondieron que sí; la P19. ¿Su universidad ofrece servicios

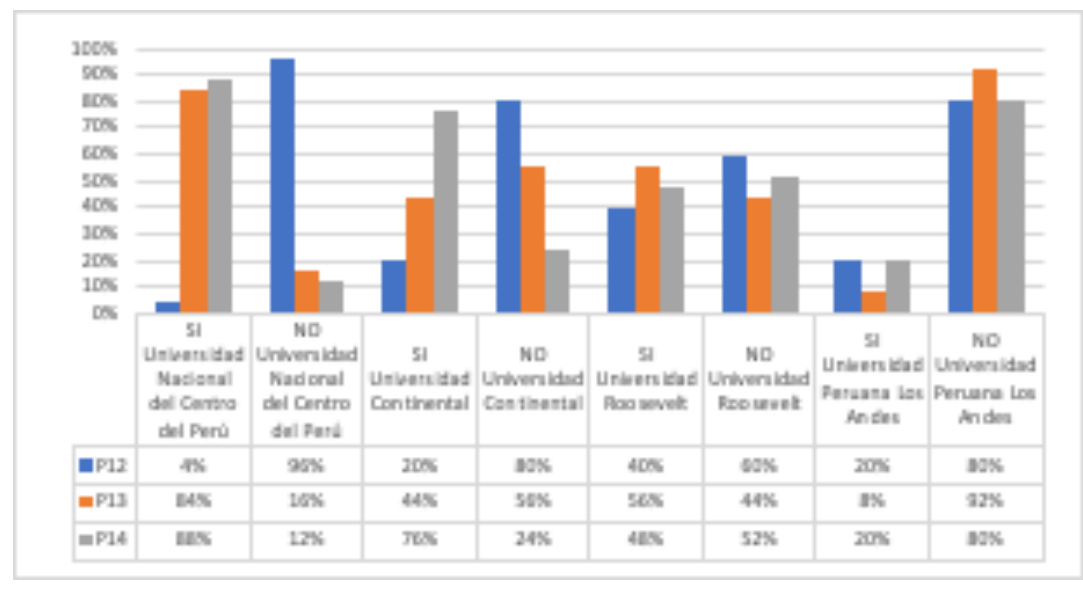

Nota. Dimensión 5, ítems de 12 a 14.

Gráfico 5. Análisis de la Dimensión 5 (D5) Docentes calificados

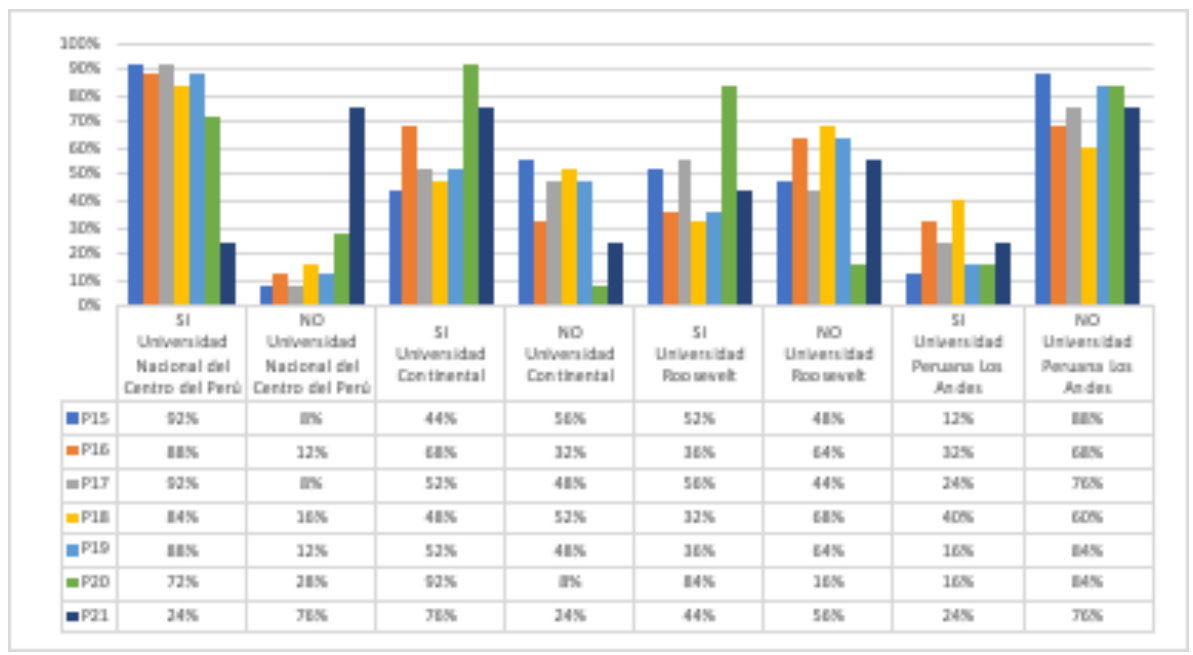

Nota. Dimensión 6, ítems de 15 a 21.

Gráfico 6. Análisis de la Dimensión 6 (D6) Servicios complementarios 
culturales (salas de lectura, participaciones de música, canto tradicional, etc) de manera virtual? 52\% respondieron que no; en la P20. ¿Su universidad realiza actividades o acciones para el cuidado y protección del medio ambiente? el 66\% de los estudiantes respondió que su universidad si está realizando acciones para el cuidado y protección del medio ambiente; en la P21. ¿Utiliza servicios de biblioteca virtual de su universidad? 58\% de los estudiantes no utiliza la biblioteca virtual. Los estudiantes universitarios no ven de una manera total y concreta la realización de los servicios complementarios que brindan soporte a su educación, es menester encontrar las estrategias óptimas que muestren una visibilidad virtual de estos indicadores (gráfico 6).

Análisis de la Dimensión 7 (D7) Bolsa de trabajo

Se analiza los resultados de la P22. ¿Su universidad cuenta con mecanismos de mediación e inserción laboral (prácticas pre profesionales y profesionales) para estudiantes y egresados? $63 \%$ ha percibidos estos mecanismos de mediación e inserción laboral; y la P23. ¿Su universidad cuenta con alianzas para la inserción laboral en instituciones públicas o privadas? $66 \%$ afirman sus respuestas corroborando que existen alianzas y convenios con instituciones públicas y privadas (gráfico 7).

\section{Análisis de la Dimensión 8 (D8) Transparencia}

En la Dimensión 8 se plantearon dos interrogantes respecto a la transparencia en las universidades.

Para conocer respecto a la siguiente dimensión se analiza la P24. ¿Conoce la página de transparencia de su universidad?, 67\% manifiesta que no; la P25. ¿Accede Ud. a la información administrativa, económica y financiera de su universidad? $73 \%$ fortalece el mismo fenómeno de la negatividad.

Claro está que en las universidades de la región Junín existe cierta carencia de comunicación respecto a la transparencia de sus actividades, queda fortalecer las plataformas de información y mejorar la socialización de la página de transparencia, gestión administrativa, económica y financiera (gráfico 8).

Análisis de la Dimensión 9 (D9) Satisfacción

Esta dimensión no se encuentra dentro de los estándares de SUNEDU, pero resulta importante conocer el grado de satisfacción que presentan los estudiantes frente a la prestación del servicio educativo, para ello se han planteado cinco interrogantes.

Respecto a la P26. ¿Está satisfecho con el servicio administrativo que le brinda su universidad? el 55\% de los estudiantes manifiestan su insatisfacción; en la P27. ¿Está satisfecho con el servicio académico que le brinda su universidad? $64 \%$ se encuentra satisfecho con el servicio; en la consulta P28. ¿Está satisfecho con el servicio de investigación formativa que le brinda su universidad? 63\% está satisfecho; en la P29. ¿Está satisfecho con la responsabilidad social y extensión universitaria que brinda su universidad? $60 \%$ manifiesta satisfacción; y finalmente en la P30. ¿Considera que está recibiendo una educación de calidad?, considerada prácticamente el resumen de toda la información solicitada, frente a ello respondieron encontrarse satisfecho en un 59\% (gráfico 9).

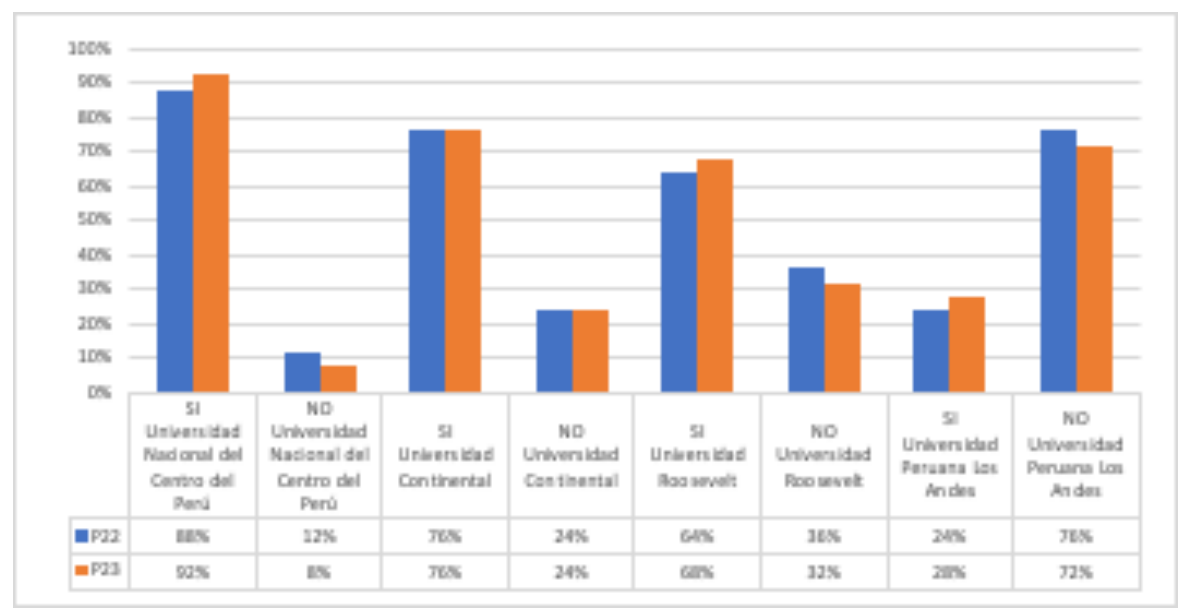

Nota. Dimensión 7, ítems de 22 a 23.

Gráfico 7. Análisis de la Dimensión 7 (D7) Bolsa de trabajo 


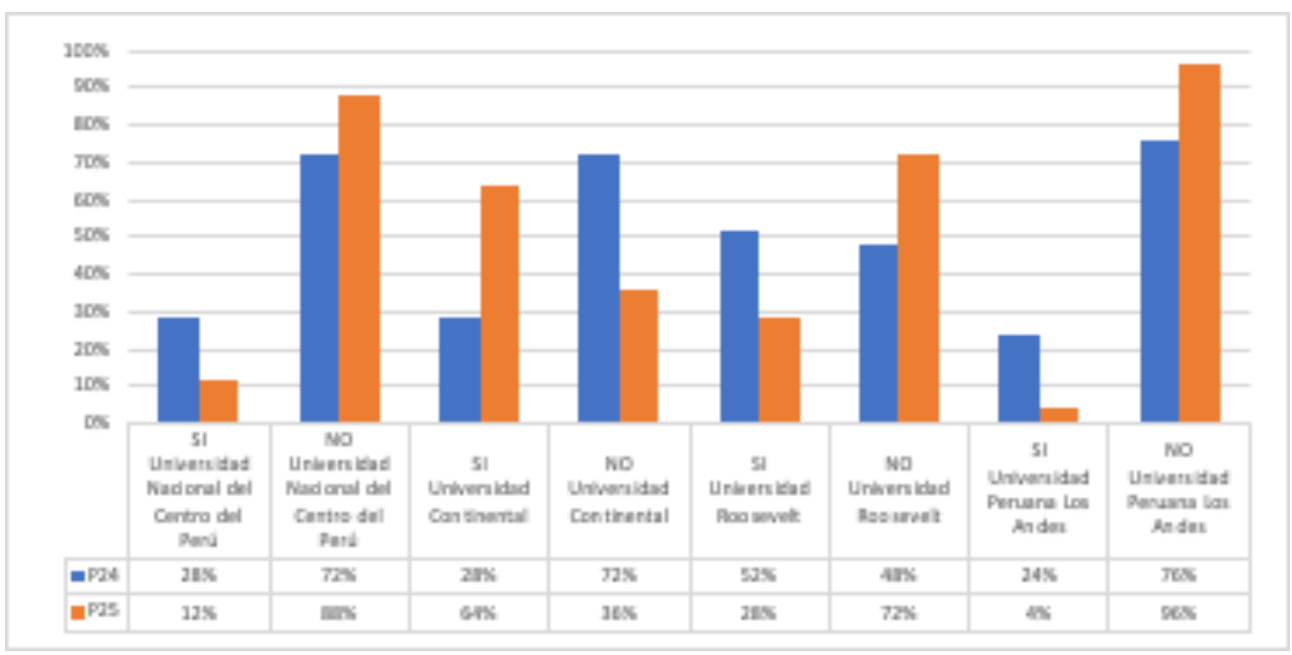

Nota. Dimensión 8, ítems de 24 a 25.

Gráfico 8. Análisis de la Dimensión 8 (D8) Transparencia

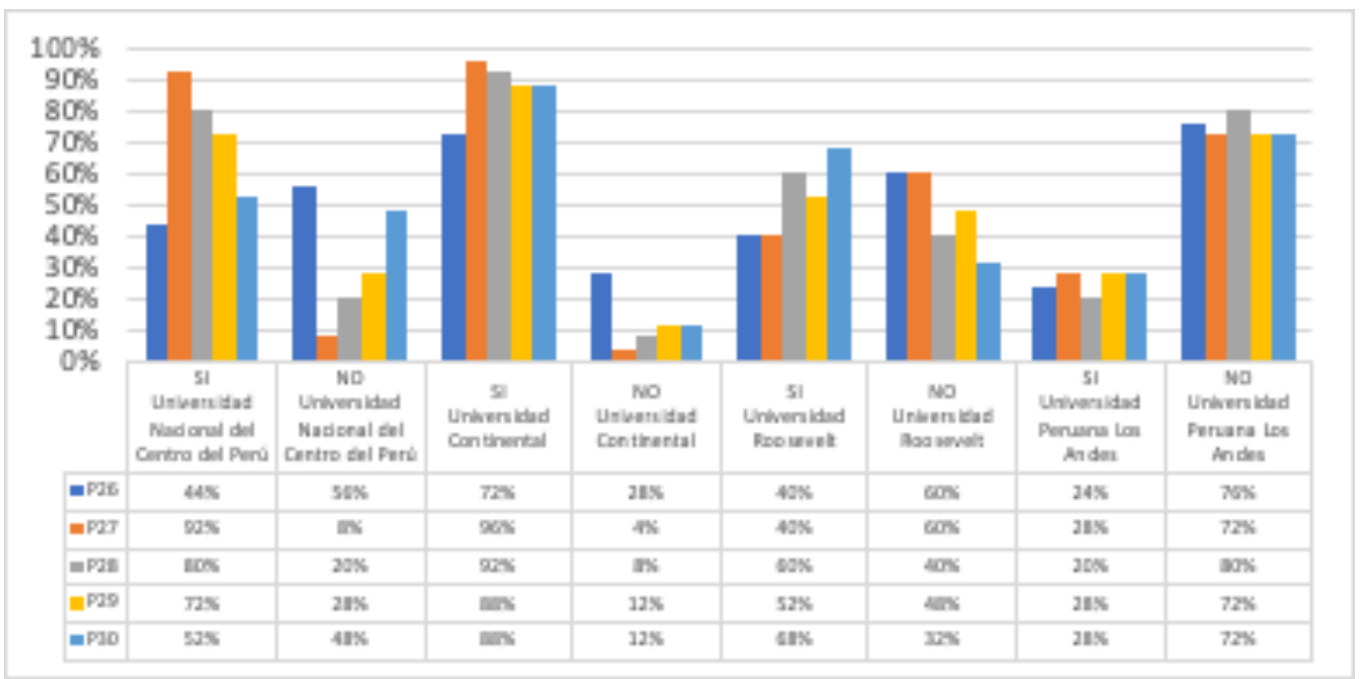

Nota. Dimensión 9, ítems de 26 a 30.

Gráfico 9. Análisis de la Dimensión 9 (D9) Satisfacción

\section{DISCUSIÓN}

La evaluación de la calidad no se puede realizar con subjetividades, se coincide con Manrique et al., cuando manifiestan que son diversos los indicadores a medir; los mismos que se relaciona directamente con la satisfacción del cliente, en este caso del estudiante (19). La medición de la percepción de la calidad, en resumen, muestra respuesta positiva en un 54\%; es decir poco más de la mitad se encuentra satisfecha con la calidad de educación brindada en tiempos de pandemia, no muy distante de los $46 \%$ de estudiantes que se encuentra insatisfecho.
Según Gil Villa et al., la educación pública no se encontraba preparada para brindar una educación virtual, generando deserción estudiantil y reclamos excesivos por la falta de calidad (20); al igual que Segovia y Said-Hung, los factores de calidad en tiempos de pandemia se han disminuido, reflejándose en una insatisfacción estudiantil (21).

Para Guadarrama-González, es importante escuchar al estudiantado; la investigación ha permitido reflejar la voz de los estudiantes y sus principales preocupaciones para el desarrollo de su profesión, sin manipulación de ningún tipo (5). Su nivel de 
satisfacción debe ser medido en tiempos promedios, con la presente investigación se pretende involucrar al estudiante de forma transversal en todos los indicadores de calidad impuestos por SUNEDU.

\section{CONCLUSIONES}

La medición de calidad es principalmente cualitativa, sin dejar de lado que esta información se puede traducir a índices cuantitativos, por ello es importante preparar una herramienta de gestión de la calidad que brinde un soporte al trabajo administrativo y docente, respaldado por el estudiante. La situación de pandemia ha generado cierto desnivel en los índices de calidad de las distintas universidades de la región Junín, reflejando esto en la insatisfacción de sus clientes internos y externos.

Los estudiantes de las universidades con licencia institucional de la región Junín, conocen los objetivos académicos de sus instituciones, pues cuentan con la aprobación de la mayoría de ellos. En la dimensión de oferta educativa muchos de ellos no cuentan con información suficiente. La infraestructura y mantenimiento, no se ha logrado suplir la prestación de estos servicios de forma virtual, en su totalidad, generando disconformidad y de manera más pronunciada en el uso de laboratorios. Para los estudiantes universitarios los servicios complementarios no otorgan soporte suficiente para el desarrollo de su carrera profesional.

La investigación en estudiantes y docente es de interés general, se conoce parcialmente el planteamiento y ejecución de proyectos de investigación y la gran mayoría ha visitado los repositorios de sus universidades. La carencia de empleo ha despertado el interés de estudiante en conocer si sus instituciones poseen estrategias de inserción laboral y convenios con entes públicos y privados. La falta transparencia es una percepción inevitable en los estudiantes, queda mucho por mejorar en los canales de comunicación. Por lo pronto poco más de la mitad de los estudiantes universitarios de la región Junín se encuentran satisfechos con la educación virtual brindada en tiempos de pandemia; queda escuchar los argumentos de insatisfacción del resto, para mejorar e implantar propuestas estratégicas que den solidez a una educación de calidad.

\section{Correspondencia}

Ketty Marilú Moscoso-Paucarchuco

Correo electrónico: cpcmoscoso@hotmail.com

\section{REFERENCIAS}

1. Clavijo-Cáceres D, Balaguera-Rodríguez AY. (2020). La calidad y la docencia universitaria: algunos criterios para su valoración. Revista de Investigación, Desarrollo e Innovación. 2020; 11(1): 127-139. DOI: 10.19053/20278306.v11.n1.2020.11688

2. Superintendencia Nacional de Educación Superior Universitaria. Registro de universidades licenciadas. Lima: Superintendencia Nacional de Educación Superior Universitaria; 2020. (Citado el 14 de diciembre del 2020). Disponible en: https://www. sunedu.gob.pe/avances-licenciamiento/

3. Cevallos D. La Calidad Educativa en la realidad Universitaria Peruana frente al Contexto Latinoamericano. Flumen. 2014; 7(1): 3-8. (Citado el 14 de diciembre del 2020). Disponible en: http:// www.usat.edu.pe/files/revista/flumen/2014-I/ ponencia4.pdf

4. Díaz AR. (2015). Factores condicionantes de la calidad en la educación universitaria peruana. LEX. 2015; 13(15): 305-331.

5. Guadarrama-González P. Calidad en la educación superior cubana: la Universidad Central Marta Abreu de Las Villas. Revista Cubana de Educacion Superior.2021; 40(2):0-0.

6. Duque E. Revisión del concepto de calidad del servicio y sus modelos de medición. Revista Innovar. 2005;1: 64-80.

7. Gonzáles O. El concepto de la universidad. Revista de la Educación Superior; 1997; 102: 1-21. (Citado el 14 de diciembre del 2020). Disponible en: http:// materiales.azc.uam.mx/omgc/descargas/CONCEP2. pdf

8. Aguila V. El concepto calidad en la educación universitaria: clave para el logro de la competitividad institucional. Revista Iberoamericana de Educación. 2004; 1: 1-8. (Citado el 14 de diciembredel2020). Disponible en: http://ladecanjose. mex.t1/imagesnew/7/4/6/3/6/COMPETITITVIDAD INSTITUTIONAL.pdf

9. Rodríguez W. El concepto de calidad educativa: Una mirada crítica desde el enfoque históricocultural. Actualidades Investigativas En Educación. 2011; 10(1). DOI: 10.15517/aie.v10i1.10088

10. Superintendencia Nacional de Educación Superior Universitaria. Modelo de Licenciamiento y su implementación en el Sistema Universitario Peruano". Lima: Superintendencia Nacional de Educación Superior Universitaria;24/11/2015. (Citado el 14 de diciembre del 2020). Disponible en: http://repositorio.minedu.gob.pe/bitstream/handle /123456789/4565/El Modelo de Licenciamiento y su Implementación en el Sistema Universitario Peruano.pdf? sequence $=3$ \&isAllowed $=y$

11. Melo-Solarte DS, Díaz PA. El aprendizaje afectivo 
y la gamificación en escenarios de educación virtual. Información Tecnológica. 2018; 29(3): 237-248. DOI: $10.4067 / \mathrm{s} 0718-07642018000300237$

12. Torres J M.Aspectos básicos para una enseñanza E-learning de calidad en la educación superior. 3C TIC : Cuadernos de Desarrollo Aplicados a Las TIC. 2016; 5(3): 8-32. DOI: 10.17993/3ctic.2016.53.8-32

13. Ayala R. Zooming in on virtual education: biopolitics and student-centred learning. Educacion Medica. 2021, 40: 1-4. DOI: 10.1016/j.edumed.2021.01.004

14. González I B, Ramírez CA. (2018). Articulación de los sistemas de calidad, Consejo Nacional de Acreditación (CNA) y normas NTC - ISO 9001 para programas académicos de educación superior en instituciones públicas. Tendencias. 2018; 19(1): 187. DOI: $10.22267 /$ rtend. 181901.93

15. Hernándes R, Mendoza C P. (2018). Metodología de la investigación - Las rutas cuantitativa, cualitativa y mixta. Ciudad de Mexico: Editoail Mc Graw; 2018.

16. Ñaupas H, Mejía E, Novoa E, Villagómez A. Metodología de la investigación Cuantitativa Cualitativa y Redacción de la Tesis. Bogotá; Edsiciones de la U; 2014. (Citado el 14 de diciembre del 2020). Disponible en: https://corladancash. com/wp-content/uploads/2019/03/Metodologia-dela-investigacion-Naupas-Humberto.pdf

17. Martínez-Salgado C. El muestreo en investigación cualitativa. Principios básicos y algunas controversias. Ciencia e Saude Coletiva. 2012; 17(3): 613-619. DOI: $10.1590 / \mathrm{S} 1413-81232012000300006$
18. Hernández-Sampieri R. Metodología de la investigación. En: Lopez J. Ratio Formationis prenoviciado. USTA; 2019. DOI: $10.2307 / \mathrm{j}$. ctvr43hvc. 8

19. Manrique K, Arcos G, Cabrera S, Bonilla M. (2021). Vista de La pandemia y su impacto en la educación superior. El uso de la tecnología por los estudiantes de la Facultad de Comunicación y Mercadotecnia de la UAGro. Cuaderno de Pedagogía Universitaria. 2021; 18: 6-17. (Citado el 14 de diciembre del 2020). Disponible en: https://cuaderno.pucmm.edu.do/ index.php/cuadernodepedagogia/article/view/408/ 436

20. Gil F, Urchaga J D, Sánchez A. Percepciones y expectativas en el alumnado universitario a partir de la adaptación a la enseñanza no presencial motivada por la pandemia de COVID-19. Revista Latina. 2020; 78: 99-119. DOI: 10.4185/rlcs-2020-1470

21. Segovia N, Said-Hung E. Factores de satisfacción de los alumnos en e-learning en Colombia. Revista Mexicana de Investigación Educativa. 2020; 26(90): 595-621. (Citado el 14 de diciembre del 2020). Disponible en: http://www.scielo.org.mx/pdf/ rmie/v26n89/1405-6666-rmie-26-89-595.pdf

Recibido: $12 / 04 / 2021$

Aceptado: 22/06/2021 